\title{
Propédeutique à la théorie des Systèmes Nationaux d'Innovation appliquée au développement économique
}

\section{Propaedeutics to the Theory of National Innovation Systems Applied to Economic Development}

\author{
Vanessa Casadella ${ }^{1}$, Dimitri Uzunidis ${ }^{2}$ \\ ${ }^{1}$ Université Picardie Jules Verne, Laboratoire CRIISEA, Réseau de Recherche sur l'Innovation (RRI), \\ vanessa.casadella@u-picardie.fr \\ ${ }^{2}$ Réseau de Recherche sur l'Innovation (RRI), d.uzunidis@openscience.fr
}

RÉSUMÉ. Largement utilisé et diffusé comme concept clé au sein des organisations internationales, tout comme actuel instrument de politique économique, le Système National d'Innovation (S.N.I.) permet d'identifier les acteurs participant au processus d'innovation dans un cadre national, tout en mettant en exergue les relations d'apprentissage entre ces acteurs. Dans cet article, nous présenterons de façon didactique et synoptique la genèse du concept, son évolution d'un point de vue académique et son application dans le cadre d'une économie en développement.

ABSTRACT. Widely used and disseminated as a key concept within international organizations, as well as a current economic policy instrument, the National Innovation System (NIS) makes it possible to identify the actors involved in the innovation process in a national context, while highlighting the learning relationships between these actors. In this article, we will present, in a didactic and synoptic way, the genesis of the concept, its evolution from an academic point of view and its application in the context of a developing economy.

MOTS-CLÉS. Innovation, Système national d'innovation, Capacités d'innovation, Développement, Politiques d'innovation.

KEYWORDS. Innovation, National Innovation System, Capacities of Innovation, Development, Innovation Policies.

Largement utilisé et diffusé comme concept clé au sein des organisations internationales, tout comme un instrument privilégié de politique économique, le Système National d'Innovation (S.N.I.) permet d'identifier les acteurs participant au processus d'innovation dans un cadre national, tout en mettant en exergue les relations d'apprentissage entre ces acteurs. Le S.N.I. décrit les relations entre institutions, privées et publiques - scientifiques, technologiques, industrielles, commerciales, financières, politiques. Ces institutions sont les entreprises, les laboratoires de recherche et de développement $(\mathrm{R} \& \mathrm{D})$, les services d'ingénierie, les administrations, etc. Les relations sont quant à elles constituées le plus souvent de flux financiers, informationnels ou humains et leur fécondation mutuelle permet de produire des innovations (nouvelles organisations, nouvelles marchandises et processus, nouvelles ressources : nouvelles combinaisons de ressources productives) dans un objectif de croissance et de développement économique durable.

Plusieurs éléments caractérisent le S.N.I. Tout d'abord, l'étude d'un S.N.I. ne s'arrête pas à l'analyse des seules conditions et stratégies entrepreneuriales de mise sur le marché des biens et services nouveaux. Les aspects éclectiques de la diffusion des innovations, les interactions entre marché, production, consommation, organisation et $\mathrm{R} \& \mathrm{D}$, les potentiels d'absorption et d'adaptation intra et inter sectoriels, les liaisons industrielles et financières, déterminent pour une grande partie, l'efficacité d'un Système National d'Innovation. L'innovation est en effet introduite dans l'économie de façon discontinue et prend de l'ampleur au fil de son adoption par un nombre important d'entreprises, de branches, de filières de production et, plus généralement, par d'écosystèmes particuliers d'activités. Si l'on suit le raisonnement de Schumpeter [11,42], les innovations se diffusent par grappes (selon les différentiels de profit qu'elles provoquent) et 
contribuent à l'évolution cyclique de l'économie. Par ailleurs, l'innovation et sa diffusion par grappes ne sont pas dues au hasard. Le contexte économique, social et politique contribue fortement à la sélection des voies de recherche et de ses applications. La compétitivité industrielle d'une économie nationale, mesurée par sa capacité à engendrer des grappes d'innovations technologiques conduisant à la formation d'écosystèmes sectoriels d'innovation, dépend fortement des liaisons interactives entre tous les acteurs du progrès technique. Enfin, l'importance du concept se trouve dans la nature systémique des liens formels et non formels entre acteurs. Les compétences, les capacités, la demande, les finances et les institutions sont autant d'éléments, qui, de par leurs mécanismes interactifs, façonnent la dynamique technologique.

Revenons sur la genèse du concept, ainsi que sur son évolution d'un point de vue académique qui a fait l'objet de nombreux travaux depuis une trentaine d'années. Le pluralisme conceptuel du S.N.I. est relatif à l'esprit de l'approche eu égard aux nombreuses déclinaisons terminologiques réalisées autour de ce dernier. Dès lors, loin d'être un concept stable et unifié, la diversité et la large envergure de l'approche sont globalement considérées comme de réels avantages. Ce sont en effet par leurs tendances convergentes et divergentes que les S.N.I. continuent d'avoir un sens [NIO, 93] et ce, aussi bien dans les économies industrialisées que dans les économies en développement.

Dans cet article, par une démarche didactique et construite à partir d'une revue de la littérature des économistes fondateurs de la théorie du S.N.I., nous présenterons en deux parties les éléments notoires que les chercheurs en économie industrielle et de l'innovation prennent en compte pour définir un système national d'innovation, puis nous appliquerons ce concept à l'économie du développement. Dans la première partie, après avoir dessiné la définition d'un système d'innovation, nous nous intéresserons aux grands traits de la " nationalité » d'un tel système. Référence sera faite aux mécanismes de régulation et de l'évolution d'un tel système régi par les règles, normes et politiques appliquées dans un pays donné. Les acteurs (entreprises, entrepreneurs, institutions de réalisation et de valorisation marchande de la recherche scientifique, etc.) combinent leurs efforts pour « produire » de l'innovation. Les incitations publiques et de l'intervention directe de l'Etat organisent et orientent ce système. En est-il ainsi dans les économies moins performantes et «en développement»? En recourant aux acquis théoriques de l'économie du développement, nous nous focaliserons sur les enjeux et les inadéquations concernant les activités (innovatrices ou non) des acteurs enrôlés dans les processus habituels de valorisation de capitaux en vue de produire, de commercer et... d'en profiter. Les failles révélées dans la conception d'une systémique de l'innovation dans un pays en développement sont dues aux relations asymétriques entre les acteurs et entre les acteurs et le - supposé - système. Nous le montrerons dans la seconde partie.

\section{De la systémique de l'innovation au Système National d'Innovation}

\subsection{La prise en compte des institutions dans la théorie économique}

Le concept de système d'innovation s'est construit par un ensemble d'approches incorporant des éléments institutionnels et évolutionnistes dans l'étude sur les changements technologiques et dans les analyses sur la structuration des systèmes scientifiques. La référence aux théories évolutionnistes dans l'approche S.N.I. a directement été posée dans les premiers travaux de R. Nelson : «dans les pays capitalistes, le changement technique est fondé sur un processus évolutionniste » [NEL, 88, p.313]. Par ailleurs, les travaux sur les systèmes d'innovation ont le mérite de sortir de la vision néoclassique traditionnelle de l'innovation comme apparaissant d'une façon quasi autonome du contexte économique sous l'influence des progrès d'une science soumise à ses propres règles. Le système d'innovation ne peut jamais atteindre

un stade optimal et d'équilibre en raison des processus d'apprentissage sujets à de continuels changements, non déterminés et dépendants de sentiers de développement. Il s'établit comme un véritable système dynamique complexe. Dès lors, cette analyse intègre l'innovation dans l'économie 
et la société en allant au-delà d'une détermination qui verrait dans l'innovation le résultat d'un investissement à but lucratif de la part d'entrepreneurs plus ou moins isolés.

En dépit de différences dans la méthode des démarches empiriques, les travaux fondés sur la systémique de l'innovation ont des caractéristiques communes essentielles : ils visent tous à approfondir la question de l'innovation comme mécanisme de création de valeur et vecteur de compétitivité. Le concept de système d'innovation offre un cadre particulièrement riche et flexible pour interpréter la diffusion des technologies, pour procéder à des analyses comparatives et pour mieux comprendre le développement des milieux innovateurs. Toutefois, le concept de système d'innovation ne peut se limiter à expliquer la manière dont la nature des interactions entre institutions existantes conditionne les trajectoires d'innovation. On cherche davantage à comprendre comment la dynamique d'innovation conduit à la formation de nouvelles institutions et formes de diffusion du savoir.

Les institutions et organisations sont d'ailleurs au cœur de l'approche. Chaque institution joue un rôle spécifique au sein du S.N.I. par la distribution de procédures conformes et de pratiques standards structurant les rapports entre individus. Ces institutions créent simultanément ordre et continuité tout en ayant un impact sur la conduite et la performance d'un système d'innovation. Les organisations, quant à elles, se trouvent enchâssées au sein d'un environnement institutionnel spécifique et se définissent comme des systèmes structurés et institutionnalisés construits pour exécuter un certain nombre de tâches. Elles représentent entre autres, les entreprises, centres de recherches et universités. Les interactions interinstitutionnelles et organisationnelles ne dépendent pas uniquement de la capacité d'innovation de l'entreprise, mais également de la capacité de son environnement et de ses partenaires externes à créer des opportunités d'innovation et à accompagner cette innovation [TEE, 07]. Par exemple, les rapports entre entités formelles (entreprises, laboratoires) et les sources informelles de création de valeur (collectifs, communautés d'usagers, etc.) contribuent à la vivacité d'un système d'innovation. L'enjeu pour les entreprises est celui de la création de valeur réalisée à l'extérieur de la firme en vue de capter la plus-value générée par le système d'affaires [TOR, 15]. Au final, les approches en termes de système d'innovation s'appuient sur trois fondements conceptuels :

i) La prise de décisions économiques repose sur des bases institutionnelles qui définissent les « règles du jeu économique » et qui conditionnent le comportement des agents et influencent leurs performances. Ces institutions évoluent sans prédétermination et sans cadre institutionnel optimal. Elles sont spécifiques et leurs configurations dépendent des contextes politiques, économiques, culturels et sociaux. Elles sont donc difficilement transférables d'un pays à l'autre.

ii) Le processus de changement se produit dans une direction sélectionnée se trouvant renforcée par des effets de rétroaction et de réponses adaptatives. La dépendance de sentier (path-dependency) ou l'historicité est l'expression d'un phénomène en relation avec la sélectivité du changement. Suivant cette dernière, le S.N.I. dépendra du chemin parcouru pour atteindre l'état final.

iii) Les connaissances technologiques sont engendrées par l'apprentissage interactif, ce qui donne naissance à des «bases de connaissances » différentes selon les agents. Ces bases communes donnent lieu à l'émergence d'opportunités d'innovation [SMI,00]. Ce dernier fondement introduit donc l'idée du mécanisme d'interactivité rejetant l'hypothèse de la séquentialité unidirectionnelle des processus d'apprentissage. Les mécanismes d'apprentissage résultent de la connectivité entre ceux bénéficiant d'idées nouvelles et qui cherchent à étendre leurs possibilités d'application et ceux qui les utilisent et manifestent des besoins continuellement révisés.

L'innovation est ici représentée en tant que « fait social », tout en dépassant le cadre stricto sensu de la technologie. Dans cette perspective, Lundvall [92] a distingué deux conceptions différentes des systèmes d'innovation : une conception étroite qui se limite aux domaines de la science, de la 
recherche, de la technologie et dans certains cas de l'éducation ; une conception large qui s'étend à tout le contexte économique et institutionnel au sein duquel émerge et se diffuse l'innovation par grappes successives. La conception étroite se focalise sur le système scientifique et technologique. Elle considère comme exogènes les sources, les causes et les conséquences d'une telle ou telle innovation technologique. L'avantage de cette approche restrictive est un gain de précision dans l'analyse du progrès technique. La littérature qui se rattache à la conception étroite [NEL, 93] fournit des études détaillées de l'architecture institutionnelle et organisationnelle des systèmes d'innovation, ou des études centrées sur l'entreprise et son environnement local : interactions entre fournisseurs et clients, entre capital et travail, etc. La conception large d'un système d'innovation permet d'étudier aussi bien les actions menant à l'innovation, que les incitations à innover, les impacts socio-économiques et institutionnels de l'innovation ou encore les effets sur le développement économique. Nous y reviendrons par ailleurs.

\subsection{La genèse du système national d'innovation}

Le concept de Système National d'Innovation (S.N.I.) a été largement utilisé depuis la fin des années 1980 pour analyser la spécificité des dynamiques institutionnelles nationales afin de créer et diffuser de nouvelles technologies. Issue de l'intuition de F. List (1841) qui s'écarte de la perspective proposée par Smith centrée sur l'échange et l'allocation de ressources, l'analyse du S.N.I. met en avant la création collective de ressources technologiques dans un espace national. Cette analyse a pris son véritable essor à la fin des années 1980 et au début des années 1990 [FRE, 87,88; LUN, 85,88,92, NEL, 88,93; NIO, 93]. Elle a connu un grand succès dans l'explication des performances nationales en matière d'innovation. Elle présente l'environnement national d'innovation (défini par les règles et normes d'accumulation applicables à tout acteur établi dans un pays donné) dans lequel les entreprises mettent en œuvre leurs stratégies technologiques et leurs processus d'innovation.

Plusieurs auteurs ont contribué à la genèse et l'affinement du concept (Tableau 1). L'analyse du S.N.I. est pour la première fois introduite de manière explicite par Freeman [87] qui identifie les causes des performances de l'économie japonaise. Il définit le S.N.I. comme " le réseau des institutions dans les secteurs publics et privés dont les activités et les interactions initient, importent, modifient et diffusent les nouvelles technologies ». Freeman avance, à travers le concept S.N.I. dans un ensemble de travaux [FRE, 87,88,95], l'idée que l'innovation technologique doit comprendre le contexte socio-institutionnel dans lequel elle s'insère et que les pays ont différents types d'adaptations institutionnelles qui s'associent aux changements des paradigmes sociotechniques. Mais ses travaux proposent une analyse générale des S.N.I. qui reste encore assez éloignée de l'analyse évolutionniste. A la suite de Freeman, le concept de S.N.I. est repris et utilisé par différents auteurs. Ainsi, le travail de Nelson [93] s'inscrit dans une définition étroite du S.N.I. et a pour objet l'étude du système technologique et des politiques technologiques de quatorze pays répartis selon les niveaux de revenu par habitant. Ne cherchant pas à dessiner un cadre théorique précis, ce travail s'attache à décrire l'évolution des activités de R\&D en repérant les sources et les montants des financements, le rôle des universités et des politiques publiques et les caractéristiques des entreprises selon les branches industrielles. L'analyse de Nelson tend à minimiser l'importance des institutions qui ont une influence macroéconomique au profit de déterminants strictement scientifiques et technologiques territorialement plus limités. Son analyse met aussi l'accent sur les interactions des agents innovateurs et les relations entre ces agents et l'environnement technologique. Du point de vue de l'analyse microéconomique, cette proposition repose explicitement sur la base du modèle évolutionniste de l'innovation.

D'un autre côté - et dans une conception plus large [LUN, 92] - un système d'innovation « est constitué d'éléments et de rapports qui interagissent dans la production, la diffusion et l'utilisation de nouvelles connaissances économiquement utilisables ». Le S.N.I. est ainsi défini comme "un système social dynamique dont l'activité centrale est l'apprentissage et la reproduction de 
connaissance au niveau du territoire d'un Etat-nation ». L'auteur place les interactions entre les producteurs et les utilisateurs de technologies au centre du système. Ces interactions reflètent non seulement les relations de marché mais aussi un contexte politique et social plus large. Par conséquent, le S.N.I. comprend l'ensemble de la structure institutionnelle d'un pays qui un rôle clé dans les processus d'apprentissage et la production et reproduction des connaissances. Cette vision est fondée sur une logique évolutionniste en soulignant le nombre important de réseaux et de collaborations entre les composantes du système. Sur un plan macroéconomique, cette définition inclut toutes les institutions politiques, sociales, économiques et culturelles affectant l'apprentissage, la recherche et les activités d'exploration scientifique : le système financier, les politiques monétaires, l'organisation interne des firmes privées, le système éducatif pré-universitaire, le marché du travail, etc. Sur un plan microéconomique, la force du S.N.I. réside plus sur l'efficacité des réseaux des firmes, les forces informelles et sources de l'apprentissage interactif au sein des activités d'acquisition, de production et de vente, que sur les activités de R\&D.

\begin{tabular}{|l|l|l|l|}
\hline AUTEUR & CONTEXTE ETUDIE & UNITE D'ANALYSE & \multicolumn{1}{|c|}{$\begin{array}{c}\text { STRUCTURE } \\
\text { ANALYTIQUE }\end{array}$} \\
\hline FREEMAN C. (1987) & Japon & $\begin{array}{l}\text { Adaptation socio- } \\
\text { économique }\end{array}$ & $\begin{array}{l}\text { Rôle du ministère du } \\
\text { Commerce International } \\
\text { et de l'Industrie } \\
\text { (Ministry of }\end{array}$ \\
& $\begin{array}{l}\text { International Trade and } \\
\text { Industry : MITI), } \\
\text { institutions éducatives } \\
\text { et de formation, } \\
\text { institutions de R\&D } \\
\text { dans l'importation de } \\
\text { technologies, structure } \\
\text { de l'industrie }\end{array}$ \\
\hline LUNDVALL B. (1992) & Pays scandinaves & $\begin{array}{l}\text { Apprentissage interactif } \\
\text { entre producteurs et } \\
\text { utilisateurs }\end{array}$ & $\begin{array}{l}\text { Rôle du secteur public, } \\
\text { éducation, institutions } \\
\text { en R\&D, institutions de } \\
\text { formation, systèmes de } \\
\text { production, marketing } \\
\text { et finance }\end{array}$ \\
\hline NELSON R. (1993) & $\begin{array}{l}\text { 15 pays développés et } \\
\text { en développement }\end{array}$ & $\begin{array}{l}\text { Cadre technologique et } \\
\text { organisationnel }\end{array}$ & $\begin{array}{l}\text { Allocation des activités } \\
\text { en R\&D, sources des } \\
\text { ressources financières, } \\
\text { caractéristiques des } \\
\text { firmes, rôle des } \\
\text { industries, universités et } \\
\text { politiques } \\
\text { gouvernementales }\end{array}$ \\
\hline
\end{tabular}

Source : auteurs

Tableau 1. Les différentes conceptualisations du S.N.I

$\mathrm{Au}$ final, le S.N.I. est un concept dynamique, ouvert aux changements de son environnement et dépendant de l'état du monde au moment de l'analyse et évoluant au cours du temps. Ce concept 
doit être apprécié en fonction de l'histoire et de ses environnements nationaux spécifiques. Ainsi, les S.N.I. se définissent dans des termes évolutionnistes [BEL,96] où ces derniers changent sous l'effet des flux qui leur viennent de leur environnement. Les changements dans les S.N.I. peuvent être lents et progressifs mais aussi discontinus et multi-stables. Ils sont caractérisés par la dépendance de sentier qui s'inscrit dans les procédures d'évolution ; les transitions sont irréversibles : il n'est souvent plus possible de retourner à des états antérieurs du système.

Si la conception de l'innovation fait l'objet de nombreux débats au sein de l'approche, les spécificités nationales et systémiques, sans lesquels le S.N.I. n'aurait pas lieu d'être, sont, en toute évidence, communément admis [FAG, 15]. Ceci nous conduit à insister sur le rôle des acteurs innovants, leurs fonctions et les liens qui existent entre ces derniers pour analyser ce concept. En fait, ces éléments clés sont ceux que la plupart des théoriciens du S.N.I. évoquent dans leurs conceptions respectives, même s'ils ne leur accordent pas tous la même importance.

\subsection{Les acteurs du système national d'innovation et leurs collectifs}

Si le S.N.I. se déploie et prend forme sur une échelle nationale, il trouve indéniablement une cohérence à partir de mécanismes d'apprentissage collectifs. Sans dispositifs collectifs, il est difficile d'accepter l'existence d'un système d'innovation. En effet, la raison d'être d'un S.N.I. réside avant tout dans le fait que l'innovation est un processus interactif dont la portée dépend du type de relations entre les différentes entreprises, organisations et comportements institutionnels dans la production, la diffusion et l'utilisation de nouvelles connaissances. Ces processus sont générés par des acteurs spécifiques que Lundvall a particulièrement décrits [85,88,92]. Selon celui-ci, les acteurs du S.N.I. sont a priori des acteurs privés qui agissent librement et spontanément en raison de leurs intérêts particuliers à l'intérieur de frontières nationales. Leurs relations prennent la forme de transferts et d'échanges de technologies dans les limites nationales. Les grandes entreprises, à travers lesquelles la science est domestiquée pour devenir une force de production, sont les acteurs clés du S.N.I. Elles jouent le rôle de pivot du S.N.I. Mais cette conception reste réductrice par rapport à la réalité, même si l'auteur souligne l'importance des innovations sociales et de la dynamique des petites entreprises innovantes dans le processus d'innovation technologique.

A l'instar de travaux de Lundvall [85], Freeman [87,88] élargit le spectre des acteurs du S.N.I. En soulignant avec insistance le rôle du gouvernement japonais dans le processus d'innovation technologique mis en œuvre par les entreprises, il met en lumière les rôles de l'Etat, des laboratoires et des institutions publiques dans la formation et l'évolution du S.N.I. Cette vision suffisamment large en termes d'intervenants permet de définir le S.N.I. comme un ensemble de synergies dont émergent des interactions entre les divers acteurs socio-économiques et politiques. Ces acteurs se situent à la fois dans les secteurs publics et dans les secteurs privés. Les activités et les interactions entre ces acteurs dans un S.N.I. visent à produire et à diffuser de nouvelles connaissances et technologies à l'intérieur d'un pays, démontrant la nécessité d'une organisation efficace du système.

Quant à Nelson [88], il inclut les institutions gouvernementales et publiques comme les universités, les laboratoires étatiques de $R \& D$ et les centres d'intervention du pouvoir public dans le S.N.I. La vision que Nelson se fait des acteurs du S.N.I. a pour particularité de reconnaître un statut privilégié à l'Etat (politique d'incitations et de financement de la R\&D) en tant qu'acteur dominant du système. Ces propositions ne sont pas contradictoires avec la définition étroite du S.N.I. de 1993 qui minimise les interventions institutionnelles et politiques dans le système, car il est évident pour l'auteur que les institutions gouvernementales et publiques, et même l'Etat, mentionnés en 1988, sont les exécuteurs importants de la recherche scientifique (surtout de la recherche fondamentale) et de la définition des incitations directes (socialisation de la production scientifique et technique au profit des entreprises) ou indirectes (financement et fiscalité favorables aux affaires, par exemple), mais ils sont de piètres gestionnaires de la $\mathrm{R} \& \mathrm{D}$ dans son ensemble qui se déroule de la genèse à la 
diffusion de l'innovation. Ce rôle revient aux entreprises et aux organisations concurrentielles de l'économie.

La vision de Nelson [88] est partiellement cohérente et concorde avec celle de Niosi et al. [93] qui affirment que l'Etat est sans doute l'acteur prédominant dans le S.N.I., même si les entreprises sont des acteurs clés du fait de la valorisation commerciale des connaissances. Niosi et ses collaborateurs soulignent les rôles de l'Etat et des institutions dans la gestion des processus d'innovation et dans les influences politiques et institutionnelles sur l'innovation. Dans le cadre national, les interactions entre les acteurs du S.N.I. se présentent comme une collaboration matérialisée par des liens portant sur les flux financiers, politiques, légaux, technologiques ou sociaux entre diverses institutions publiques ou privées. Au final, Niosi et al. montrent plus particulièrement la nécessité pour les acteurs privés d'entretenir des liens privilégiés avec les institutions publiques.

Dernière approche à laquelle nous nous intéressons (malgré d'autres travaux bien nombreux en la matière) est celle de ceux qui considèrent le S.N.I. comme « un ensemble d'institutions distinctes qui contribuent conjointement et individuellement au développement et à la diffusion des nouvelles technologies et qui forment le cadre à l'intérieur duquel les gouvernements formulent et mettent en ouvre les mesures destinées à influer sur le processus de l'innovation »[OCD,99]. Cette vision s'appuie sur le fait que les acteurs du S.N.I. sont tous ceux qui interviennent dans le processus d'innovation et dont leurs collectifs sont fondés sur la création, l'acquisition, le transfert et la diffusion de connaissances et de nouvelles technologies. Parmi ceux-ci, l'Etat est un acteur particulier qui détermine non seulement les activités des agents innovateurs mais aussi les relations entre ces derniers.

Sur le plan de la performance de l'une économie nationale, les acteurs précédemment cités construisent une dynamique d'apprentissage qui se réalise dans un pays donné et ceci malgré la libre circulation de l'information, de la connaissance, de capitaux, des biens et des services. Mais avec la globalisation des échanges, la dynamique de la régionalisation, la standardisation de la technologie et l'intensification de la compétition au niveau international, des éléments hors de la frontière nationale peuvent influencer les changements et les évolutions des S.N.I. Les comportements de recherche et d'innovation des firmes multinationales dépassent d'ailleurs les limites nationales. Ce type de firmes s'intègre à la fois dans le système d'innovation de son pays d'origine et dans les systèmes d'innovation des pays hôtes. Elles accélèrent les flux informationnels, cognitifs et financiers à travers le monde et conditionnent la dynamique d'évolution des S.N.I de départ et d'accueil. Les processus d'innovation tendent à devenir globaux, les systèmes nationaux d'innovation sont de plus en plus ouverts [LUN, 94] et l'innovation ouverte prend le pas sur l'organisation étatique de la valorisation de la recherche à des fins néomercantiles [UZU, 10].

\subsection{Les relations entre acteurs d'un S.N.I.}

Si un S.N.I. englobe une multitude d'acteurs, ces acteurs sont reliés par divers réseaux formels et informels qui réalisent des échanges commerciaux, financiers et informationnels, et qui sont influencés par la nature interactive du système. Les relations entre les acteurs du S.N.I. sont plus complexes que celles présentées par le modèle de liaison en chaîne de l'innovation [KLI,86] fondé essentiellement sur les éléments technologiques.

Dans le cadre du S.N.I., trois types de relations entre les acteurs peuvent être distingués : premièrement, les relations de nature scientifique et technologique mettent au premier plan le caractère essentiel de l'innovation. Ces relations se produisent notamment entre les acteurs dont les activités sont consacrées au progrès scientifique et technologique ou sont liées directement à l'innovation technologique. Deuxièmement, les relations de nature financière reposent sur le financement des activités d'innovation et de R\&D. Elles concernent les institutions financières et l'Etat d'une part, et les agents innovateurs engagés dans le programme de recherche d'autre part. Et 
troisièmement, les relations de nature administrative font intervenir le rôle managérial de l'Etat et des institutions publiques. Les différentes approches du S.N.I. soulignent l'importance du rôle gestionnaire de l'Etat dans la mesure où la compétitivité structurelle d'une économie nationale en dépend. Les relations, généralement de nature très diversifiée, constituent l'environnement d'innovation d'un pays en faisant appel à des compétences appropriées, de savoirs, d'expériences et de qualifications des acteurs. Les relations étroites et dynamiques entre Etat et acteurs sont déterminantes pour la caractérisation de la nature et de la performance d'un S.N.I.

Le S.N.I. peut être ainsi illustré dans la figure suivante dans laquelle les acteurs principaux et leurs relations interactives sont mis en évidence :

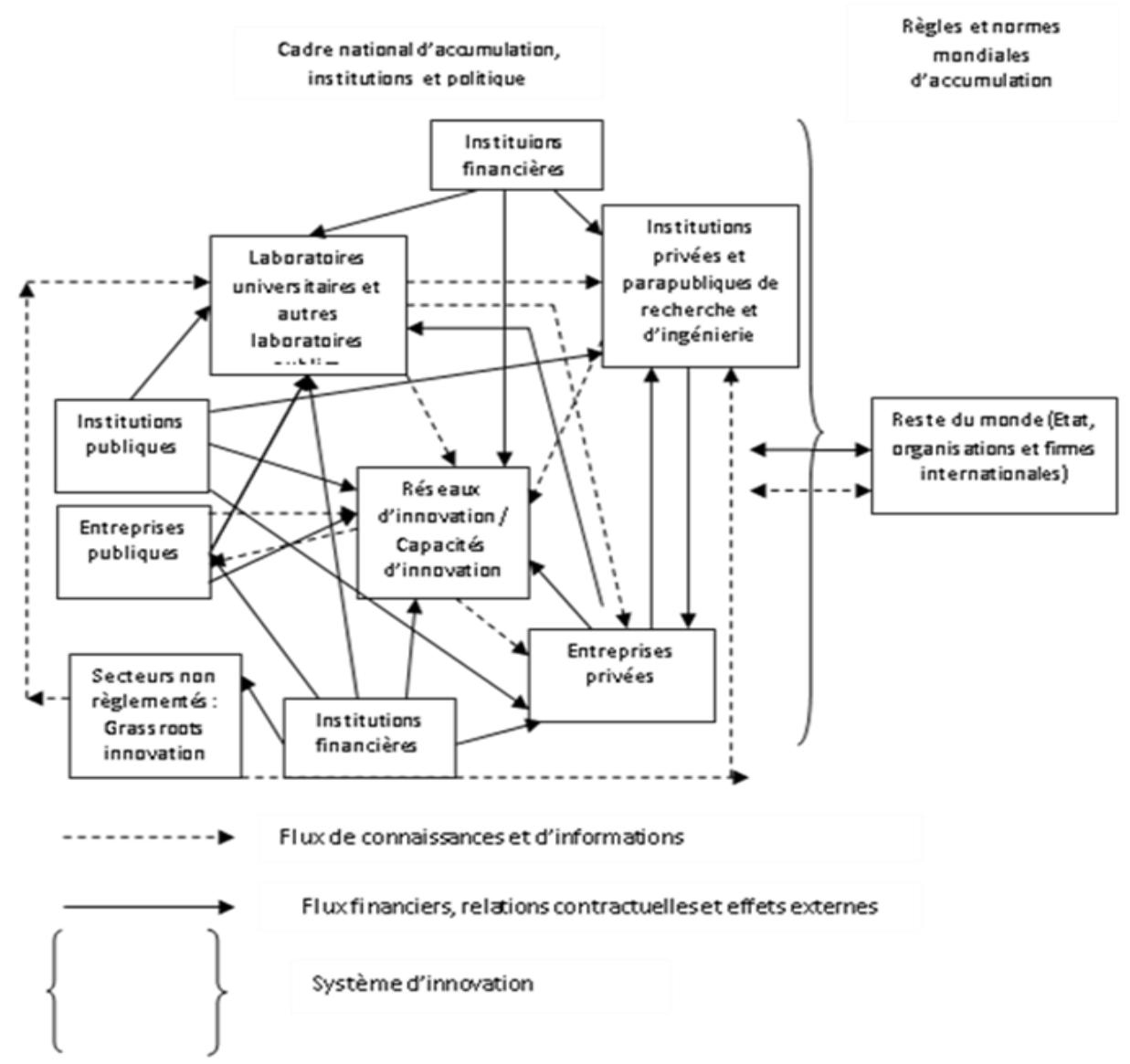

Graphique 1. Le S.N.I et ses interrelations

Les entreprises et les laboratoires privés, les centres de recherche et les laboratoires publics sont les acteurs les plus actifs dans le S.N.I. et ce, en raison de l'intensité des flux de connaissances, d'informations et de capitaux. Les relations entre ces acteurs sont toujours complexes mais dynamiques. L'Etat, comme précédemment mentionné, est tout aussi important car il finance et exécute souvent une grande partie de la $\mathrm{R} \& \mathrm{D}$ nationale (surtout la partie non immédiatement valorisable sur le marché), crée et pilote les organisations publiques et oriente les organisations privées. Il définit le cadre d'activités d'innovation et les règles du jeu permissives à la mise en œuvre des processus d'innovation. Par les règles de coordination et par l'application des politiques technologiques et industrielles à long terme, les interventions de l'Etat favorisent avec plus ou moins d'intensité et plus ou moins de succès les interactions entre les acteurs nationaux d'innovation. Dans cette perspective, l'environnement national des entreprises est à la fois défini par les acteurs principaux du S.N.I. mais aussi par leurs relations formelles et informelles. Concernant enfin l'environnement technologique des entreprises au sein duquel elles innovent, ce sont les interventions de l'Etat en matière de politique technologique qui doivent particulièrement être prises 
en compte. Dans tous les cas, l'environnement peut être appréhendé à travers le rôle de l'Etat et les outils de ses interventions dans le domaine de la recherche et de l'innovation.

\subsection{L'Etat et son intervention dans la vision évolutionniste de l'innovation}

Pour les évolutionnistes, un pays, comme une entreprise, se trouvent situés dans leur développement, sur une trajectoire technologique qui conditionne largement leurs capacités d'assimilation des nouvelles technologies. Cette trajectoire technologique est largement déterminée par le poids de leur histoire économique, l'état de l'environnement socio-économico-technique, connu imparfaitement, et le fonctionnement du marché à l'instant présent. L'approche évolutionniste met également l'accent sur la nature de l'accumulation et de l'apprentissage dans le processus d'innovation, et insiste sur les interactions complexes entre les acteurs innovateurs. Selon cette vision, les activités de R\&D, en particulier la recherche fondamentale, possèdent des potentiels commerciaux. Elles peuvent également enrichir les connaissances accumulées, améliorer les capacités d'apprentissage et renforcer les compétences des entreprises. Il y a ainsi pour les entreprises des incitations naturelles à investir dans des activités de R\&D et à s'engager dans des coopérations avec d'autres entreprises ou avec de différentes institutions pour partager et créer de nouvelles connaissances. A cet égard, l'approche évolutionniste postule implicitement que le soutien à la recherche fondamentale peut provenir aussi bien du secteur privé que du secteur public. Le fait que l'ensemble de la recherche fondamentale relève de la seule responsabilité de l'Etat n'est plus une règle absolue, même si elle reste importante.

Une des tâches du décideur public est donc de concevoir des modalités institutionnelles qui permettent une valorisation commerciale en tant qu'utilisation des travaux de recherche fondamentale à des buts lucratifs. Et ce sans compromettre la sélection des projets ou l'évaluation des travaux de recherche par les pairs [MET,94]. La recherche appliquée engagée par les entreprises peut également engendrer des externalités positives et des opportunités technologiques à travers des relations interactives. Des connaissances génériques peuvent découler autant des travaux de recherche fondamentale que des travaux de recherche appliquée et de développement. En comparaison avec le modèle linéaire d'innovation, les retombées de R\&D ont potentiellement une portée plus vaste. L'argument en faveur d'un soutien public direct à la $R \& D$ apparaît d'une manière générale plus convaincant.

Dans ce cadre, le rôle de l'Etat dans la vision évolutionniste de l'innovation ne se limite pas seulement à l'utilisation des instruments de soutien à l'innovation comme celle illustrée dans le modèle linéaire montrant une progression à sens unique allant de la recherche fondamentale jusqu'à l'innovation en passant par la recherche appliquée, le développement technologique et le prototypage $^{1}$. Il trouve sa cohérence dans des domaines beaucoup plus larges en se manifestant de façons très diverses. En effet, l'intervention de l'Etat consiste non seulement à supporter la recherche fondamentale dans les domaines publics, mais aussi à soutenir et à promouvoir ce type de recherche dans les domaines privés. L'intervention publique en matière de politique d'innovation ne se limite plus seulement au brevet, mais cherche à favoriser les coopérations et les échanges de connaissances entre différents acteurs d'innovation. On ne parle plus de «politiques d'innovation » au sens strictement technologique; la performance d'une politique d'innovation ne se mesure pas par ses effets sur la $R \& D$, mais par son véritable impact sur les relations du système. D'où les différentes complémentarités entre les éléments d'un système d'innovation et les instruments de politique économique (Mohnen, Röller, 2005). Si, dans un système d'innovation dynamique, un

\footnotetext{
${ }^{1}$ Dans le modèle linéaire de l'innovation, les instruments consistent en la prise de mesures ex ante comme, par exemple, les subventions qui impliquent un soutien financier direct et important de l'Etat aux premières étapes de l'innovation et des mesures ex post comme la propriété intellectuelle qui suppose la définition d'un cadre réglementaire cherchant l'équilibre entre des effets incitatifs contradictoires: le besoin d'un côté de protection et de l'autre côté de diffusion des connaissances scientifiques et techniques.
} 
acteur est absent ou ne parvient pas à interagir avec les autres, il peut bloquer l'ensemble du système d'innovation. On comprend donc l'intérêt de considérer la politique d'innovation comme partie intégrante de la politique économique et sociale en général.

\subsection{Les logiques de mise en œuvre des politiques d'innovation}

Dans la visée de l'analyse évolutionniste, les principes de l'intervention de l'Etat en matière de politique technologique (et d'innovation) consistent en une nouvelle gestion des externalités de connaissances d'une part, et en une nouvelle légitimité de ses interventions d'autre part. Ces deux principes constituent essentiellement la base de l'environnement technologique de l'entreprise.

Tout d'abord, l'intervention publique en matière de politique technologique se focalise essentiellement sur le problème de l'externalité des connaissances. Dans l'approche standard, ce problème peut être appréhendé à travers deux hypothèses centrales : la première concerne la définition de la connaissance, mais aussi de l'information ; la seconde concerne le fait que les seules interactions prennent place sur des marchés concurrentiels où les seules informations qui circulent et déterminent les transactions sont les prix. En remettant en question ces deux hypothèses, le courant évolutionniste propose de nouvelles orientations sur les externalités des connaissances et leurs implications sur les principes d'intervention des politiques technologiques. La reformulation de la première hypothèse consiste à distinguer la connaissance tacite des connaissances codifiées, et non plus à considérer simplement la connaissance comme une simple information. Elle implique également que le rendement social n'est pas aussi élevé qu'il le serait si les coûts de diffusion des résultats de la recherche et de l'innovation étaient négligeables; ce qui veut dire que le rendement social de la recherche est surévalué lorsque la connaissance est assimilée à l'information dont la diffusion est sans coût. La reformulation de la seconde hypothèse concerne la prise en compte des formes contractuelles variées et non plus uniquement des relations marchandes concurrentielles. Ceci implique que le rendement privé pourra être supérieur de celui prévu par la théorie standard de l'équilibre concurrentiel. Dans cette nouvelle approche, la place de l'intervention publique peut apparaître réduite puisque 1'allocation décentralisée des ressources n'est plus aussi éloignée de l'efficience sociale.

L'approche évolutionniste de l'innovation technologique, se voulant plus riche et plus réaliste, ne simplifie cependant pas la formulation de mesures de politique technologique (et d'innovation). Elle confirme en revanche l'existence de deux objectifs de politique technologique (et d'innovation) qui peuvent être d'apparence conflictuels. Ainsi, l'amélioration du rendement privé de la recherche peut se faire conjointement avec l'amélioration de son rendement social. En effet, l'objectif de l'intervention publique ne se cantonne pas à la gestion des externalités qui seraient données, mais également à la promotion de certaines entre elles, permettant une endogéneisation des externalités particulières. Ainsi, Cohendet et al. [99] soulignent que «dans un contexte essentiellement dynamique, le rôle qui semble naturellement dévolu à la puissance publique est de créer les conditions de rendement croissant et de renforcement mutuel des projets et activités d'innovation, en se focalisant sur l'organisation du système de distribution de connaissances et en développant autant que faire se peut son 'pouvoir distributif'»(p.373). A la différence de l'approche informationnelle de la technologie qui renvoie à un univers purement concurrentiel dans lequel les entreprises luttent contre les fuites d'informations (spillovers), l'organisation d'un système « riche » en externalités positives se réfère à un univers où la confiance entre les acteurs est accrue et où le degré d'ouverture et de diffusion des connaissances est important [PEN,03]. Les entreprises sont donc non seulement reliées par des relations concurrentielles, mais également par des relations de coopération dans le sens où les entreprises concurrentes mettent en place des dispositifs de coopération temporaires [NAL, 96]. Pour que cette vision apparaisse réaliste et pertinente, les interventions publiques doivent aider à satisfaire certaines conditions institutionnelles. 
Si les interventions publiques dans le modèle linéaire d'innovation impliquent une différenciation entre mesures ex ante et ex post dans l'utilisation des instruments de soutien à l'innovation, concernant la gestion des externalités de connaissances, les politiques technologiques et industrielles dans la conception évolutionniste de l'innovation envisagent les mêmes problèmes de coordination mais de façon plus complexe. De plus, les interventions publiques doivent faire face aux problèmes liés aux externalités dynamiques et aux questions d'équité qu'elles posent [NEL, 82].

Ex ante, l'intervention de l'Etat consiste non seulement en un soutien financier, mais plus particulièrement en la stimulation de la dynamique des interactions collectives qui est la source de l'externalité. La politique technologique doit constituer un environnement favorable à la formation des partenariats et des réseaux technologiques entre le secteur public et le secteur privé et à l'intérieur de chaque type de secteur. Elle doit également inciter les entreprises à prendre en charge des activités qu'elles n'ont pas suffisamment intérêt à développer elles-mêmes en raison des externalités positives.

Ex post, l'aspect fondamental de l'intervention publique est de diffuser les externalités positives à une plus grande échelle. Il s'agit d'un problème de distribution efficace des connaissances publiques aux entreprises ou aux laboratoires privés d'une part, et d'un problème de transformation d'un bien privé en un bien public ou semi-public sans diminuer les incitations des initiatives privées d'autre part. Ainsi, la politique technologique ne s'arrête pas à la garantie de la protection par les brevets, mais aussi à l'amélioration de la souplesse et la modularité du système des droits de propriété intellectuelle. Ce dernier facilite l'arbitrage des entreprises entre la sécurisation des droits de propriété des connaissances internes et l'obtention de droits d'accès aux connaissances complémentaires externes.

Par ailleurs, les interventions publiques doivent prendre en compte la question des externalités dynamiques - à long terme - proposée par Nelson et Winter [82] : «les externalités dynamiques ne sont plus susceptibles de définir pour une fois et pour toute catégorisation, elles sont plutôt liées étroitement aux contextes historiques et institutionnels particuliers » (p.368). Cette question repose sur le problème de l'écart entre les rendements privé et social en matière d'investissements liés à l'innovation. Selon les auteurs, cet écart augmente quand l'horizon temporel s'éloigne et soulève des problèmes d'équité et de relations entre les générations de connaissances. En effet, les générations futures des connaissances peuvent bénéficier de la génération actuelle, tout comme la génération actuelle peut le faire à partir des connaissances accumulées. Le marché n'est pas une institution destinée à résoudre naturellement ce type de problème, c'est à la puissance publique de poursuivre ce type d'objectif et d'assurer une redistribution intergénérationnelle des ressources en matière de recherche [GUE, $03 ;$ GOD, 04].

Pour synthétiser, la traditionnelle politique technologique axée sur le financement et la réalisation de grands programmes de $R \& D$ dans le domaine public a été partiellement substituée par une politique de recherche et d'innovation plus générale focalisée sur la gestion du S.N.I. C'est-à-dire qu'au-delà de son rôle en termes de financement, l'Etat met en œuvre des mesures incitatives permettant la coopération et la synergie entre tous les acteurs innovants pour générer des ressources d'innovation. Ces mesures conduisent également à l'amélioration de l'attractivité économique et technologique nationale.

Le rôle de l'Etat dans la régulation de la socialisation de l'innovation à des fins privées est indispensable et précis. L'intervention publique se situe initialement dans l'organisation de l'offre de ressources scientifiques à commencer par leur transfert du public vers le privé. Les politiques d'innovation rendent accessibles aux entreprises tous les moyens scientifiques de recherche, de développement, d'application et de choix technologiques pour leur permettre l'élaboration de nouveaux moyens et procédés de production, de nouveaux biens et services dans l'industrie. De même, l'Etat développe un cadre propice à la diffusion-appropriation de l'innovation par la révision 
des règles de concurrence (dont la remise en cause des situations de monopole sur les marchés des biens et services, de la finance et du travail) et l'introduction des principes du marché dans tous les domaines de l'activité économique.

La nouvelle économie de la croissance a théorisé ce processus, mettant en cause la vieille idée des «rendements décroissants», de la manière suivante: en partant de l'idée des externalités engendrées par la mise en valeur du «capital scientifique » et du «capital humain», les économistes néo-libéraux font appel à l'Etat pour prendre en charge une grande partie des coûts sociaux - liés à l'innovation. Par exemple, dans la R\&D, où le stock de connaissances ne s'amortit pas et où la production de chaque entreprise peut bénéficier de l'ensemble de la connaissance disponible ; dans la formation du «capital humain » aussi, où les qualifications, les compétences et les connaissances des uns doivent profiter aux entreprises. L'intervention étatique est primordiale dans la gestion de la socialisation, par les relations marchandes, de la production : financement des activités qui génèrent des ressources appropriables individuellement ou collectivement par les entreprises; création de mécanismes permettant la réappropriation privative du rendement de l'investissement en recherche-développement (p. ex. brevets qui ne gênent pas la diffusion des innovations); mise en œuvre de procédures de coopération entre entités publiques et privées dans le but d'assurer financièrement la faisabilité d'un projet d'investissement privé susceptible d'avoir des retombées économiques sur une large échelle [AGH, 98].

La formation d'un réservoir de capacités productives accessibles et appropriables à tout moment par les entreprises est considérée par les économistes cités ci-dessus comme l'aspect fondamental de l'intervention publique dans la formation et l'évolution d'un S.N.I. Suivons le raisonnement des Branscomb et Keller [98] : constatant que la création et la diffusion de connaissances accroît les performances d'une économie nationale (et des grandes entreprises qui la composent), ils avancent l'idée que la traditionnelle politique scientifique et technologique (axée sur le financement et la réalisation de grands programmes de recherche et de développement, essentiellement, dans les domaines de la défense, de l'énergie, de l'espace ou de la médecine) a été substituée par la politique de recherche et d'innovation. Cette politique pour être fructueuse sur le plan de la compétitivité doit viser autant la réalisation des programmes de recherche publics ou financés sur fonds publics que la diffusion aux « utilisateurs » (les entreprises concurrentielles) de leurs résultats. L'Etat doit garantir l'efficacité des procédures de privatisation (la «valorisation ») par la réglementation (protection de la propriété industrielle, lois anti-trust, etc.), la fiscalité, le budget, etc. dans le but de favoriser l'accumulation du « capital social». Par ce terme, ces économistes conceptualisent les processus de transfert de valeur d'une entreprise à une autre, des institutions publiques vers les entités privées ; ils conceptualisent aussi la formation d'un «stock» (réservoir) de ressources mises en commun lors de cette coopération multiforme, multifonctionnelle et multipartenariale et de la formation d'un écosystème d'innovation.

\section{L'application du concept de S.N.I. dans les pays en développement}

\subsection{S.N.I. et développement}

Si le concept du S.N.I. a trouvé un intérêt notoire dans les économies du Nord, les recherches sur l'innovation systémique dans les économies du Sud se sont également diffusées depuis une vingtaine d'années [KIM, 97 ; HOB, 95, AMS, 01 ; BEL, 07]. Plus récemment, la question a été de savoir si l'approche du système d'innovation représentait un instrument réellement pertinent pour appréhender la croissance et le développement des pays du Sud [LUN, 09; MUC, 13]. L'idée est d'utiliser ce concept pour comprendre les processus d'apprentissage et d'innovation des économies en développement. L'analyse du S.N.I. ne servirait donc plus d'unique instrument pour comparer les performances technologiques des Etats mais bien d'outil de promotion du développement économique. 
L'analyse du S.N.I. a progressivement gagné une forme de cohérence dans les économies du Sud caractérisées par un plus faible taux d'accumulation et une plus faible compétitivité structurelle que les pays industriels qui dominent et organisent l'économie mondiale. Mais la structure du secteur privé dans les pays en développement (P.E.D.) ainsi que ses performances différent de celles des économies du Nord [CAS, 17]. Ceci exige de considérer un certain nombre d'éléments plus spécifiques, tels que la faiblesse des systèmes juridiques (droits de propriété moins sûrs, coûts de transactions plus élevés), les différences de conditions de la demande (faible pouvoir d'achat, demande de produits plus ou moins sophistiqués), les infrastructures déficientes (coûts de transport et de production élevés), la faiblesse des systèmes éducatifs (de l'école primaire aux universités) et la plus grande volatilité des prix. Plus globalement, les approches sur les systèmes d'innovation ont besoin de prendre en compte l'ensemble des particularités propres aux P.E.D. [ALT, 09].

Dans les P.E.D., en effet, l'innovation est d'abord très relationnelle. Elle est marquée par un fort capital social. Si Lundvall [02] le rappelle déjà dans les économies du Nord, l'« innovation relationnelle » mérite une plus vive attention dans les pays du Sud [ARO, 03]. L'impact des liens sociaux sur l'innovation et l'apprentissage est important puisque l'intensité de ces liens fournit de riches sources d'information et de connaissances nécessaires aux activités d'innovation. Les liens sociaux, à travers la confiance, l'empathie, les relations affectives, sont autant de qualités permettant l'acquisition plus rapide des connaissances et la promotion de l'innovation. Dans les dynamiques de clusters industriels (écosystèmes intégrés d'innovation), Mc Cormick [99] associe dans cette même perspective l'importance du collectivisme africain avec ses influences sur l'apprentissage. L'effet de clusters, lié à la proximité géographique et à un fort capital social, encourage le partage de l'innovation et les opportunités pour l'apprentissage de nouvelles techniques.

L'innovation est ensuite représentée dans les secteurs de faibles et moyennes technologies sans se limiter aux secteurs de pointe et à la $\mathrm{R} \& \mathrm{D}[\mathrm{JOH}, 03]$. Tandis que les innovations dans les secteurs des hautes technologies sont sophistiquées et fondées sur la science et l'innovation radicale ou disruptive, l'innovation dans les économies en développement représente davantage des activités routinières d'apprentissage au sein de petites structures traditionnelles et/ou des activités d'incorporation de nouvelles technologies et méthodes dans les entreprises plus grandes insérées dans les secteurs ouverts et concurrentiels de l'économie. L'innovation est donc principalement conduite de manière informelle par les techniques d'apprentissage par la pratique, l'usage et l'interaction. Les activités de R\&D ne sont pas clairement et formellement articulées au sein de la stratégie de l'entreprise. Il est donc important de mettre en exergue la diversité des processus d'apprentissage ${ }^{2}$ dans les études sur l'innovation systémique concernant les P.E.D.

Comme nous l'avons vu plus haut, l'innovation est fortement marquée par son environnement global et est appréhendée à partir de ses trajectoires d'évolution. Le contexte de l'innovation dans les P.E.D. est souvent complexe. Les économies sont instables, vulnérables et les stratégies d'innovation sont liées à leur environnement macroéconomique, politique, institutionnel et financier [CAS, 03]. On parle de système national d'inertie [HOB, 04] dans les P.E.D. afin de mettre en relief les problèmes sociaux, politiques et techniques rencontrés par ces derniers et envisagés comme un véritable frein à l'innovation. L'hétérogénéité de ses trajectoires d'évolution est donc à considérer, comme les dynamiques de rattrapage par l'élaboration de capacités technologiques [MYT, 03].

Ensuite, les stratégies d'innovation du Sud s'interprètent à travers leur construction et non leur propre résultat technologique. Les systèmes d'innovation du Sud présentent la particularité d'être

\footnotetext{
${ }^{2}$ La diversité des processus d'apprentissage est mise en exergue dans le mode DUI (Doing, Using, Interactif) proposé par Jensen et al. (2007). Une grande partie de l'apprentissage, notamment à travers la connaissance tacite et localisée, se réfère à l'apprentissage sur le tas. Ce mode se focalise notamment sur l'apprentissage interactif à travers les institutions et les interrelations.
} 
incomplets ou déséquilibrés dans le sens où certains de leurs aspects sont inefficients ou simplement non existants [NAR, 04]. Selon les économies étudiées, on évoque les nombreuses rigidités organisationnelles et institutionnelles, l'inadaptation des politiques macro-économiques, les faibles investissements en éducation et en $\mathrm{R} \& \mathrm{D}$, ainsi que leurs faibles capacités de production et d'assimilation technique. Les systèmes d'innovation du Sud sont globalement dépeints à partir de leurs diverses défaillances systémiques [EDQ, 01]. Il devient donc difficile de parler du caractère $a$ priori (ex-post) de l'innovation lequel est utilisé afin de décrire les processus d'innovation des pays industrialisés dotés d'une forte base institutionnelle et d'une infrastructure avancée. Les stratégies d'innovation du Sud doivent plutôt revêtir un caractère a posteriori (ex-ante) afin que l'analyse sur les processus d'apprentissage puisse se déplacer plus en amont sur ses conditions d'émergence et de développement. On évoque généralement le terme de construction ou de promotion du système d'innovation du Sud [LUN,02].

Enfin, l'innovation est liée aux stratégies de développement et de réduction de la pauvreté. Dans les P.E.D., le traitement inégal des minorités et la mauvaise gouvernance locale à travers les problèmes de corruption détournent la construction du capital social et limitent automatiquement les capacités d'apprentissage de la société. L'économie de rente et la mauvaise gouvernance ne favorisent pas la création de savoirs ; la main-d'œuvre hautement diplômée n'étant pas employée dans une perspective de valorisation de ses compétences et qualifications.

L'innovation systémique n'est donc pas ici une simple question de ressources pour promouvoir la recherche technologique mais bien un ensemble d'institutions qui soutiennent l'apprentissage interactif dans un objectif, tel que conceptualisé par Sen [00], de libertés et «capabilités». Les capacités individuelles des agents économiques dépendent de supports institutionnels de la société et de ses arrangements sociaux, politiques et économiques. On évoque le terme de capacité manquante pour justifier la nécessité de créer une nouvelle voie d'entrée par la capacité d'apprentissage dans les stratégies de développement [JOH, 03]. En ce sens, la capacité technologique devient une composante essentielle du développement [JUM, 01].

Un ouvrage d'Arocena et Sutz [03] résume dans cette perspective l'esprit du Système National du Sud en comparaison avec celui du Nord (Tableau 2) :

S.N.I. du Nord :

S.N.I. du Sud :

Le S.N.I. est un concept :

Ex post, élaboré à partir de l'analyse de process réellement existants.

Ex ante, utilisé pour analyser les processus de nature essentiellement virtuels.

\section{Le S.N.I. est un concept fondé sur les relations mais :}

Les relations d'innovation sont imbriquées dans un tissu social dense.

Le tissu de relations innovantes est fragmenté et souvent plus informel que formel

Le concept de système d'innovation oriente la conception des politiques qui :

Doivent soutenir les efforts délibérés visant à renforcer le dialogue entre acteurs clés de l'innovation.

Reflètent la légitimité sociale (historiquement construite) des efforts nationaux pour engendrer la construction de savoirs et de l'innovation.
Se réduisent à une « copie » d'institutions et ne génèrent pas d'actions spécifiques valorisant amplement l'innovation existante, à savoir informelle et de nature « interstitielle».

Reflètent l'absence de consensus sur le rôle de la $R \& D$, conséquence d'un mauvais diagnostic entre l'adaptation des connaissances étrangères et la création de propres savoirs. 


\subsection{Vers un S.N.I. inclusif structuré autour de capacités et de compétences}

Le concept de «Système d'Innovation et de Construction de Compétences » (S.I.C.C.) a été présenté pour la première fois par le groupe Globelics ${ }^{3}$ en 2002 lors d'une Conférence Internationale sur les Systèmes d'Innovation à l'Université d'Aalborg. Lundvall [13] a récemment rappelé son importance dans les économies du Sud :

- La «construction de compétences» permet de comprendre comment les P.E.D. peuvent transformer la croissance économique reposant sur une forte demande de matières premières vers le développement de capacités d'innovation dans l'industrie et les services. L'assimilation des capacités basées sur la science et technologie est problématique dans ces pays.

- La « construction de compétences » permet ensuite de comprendre le rôle de l'ingénierie et des capacités d'innovation dans le développement économique. La construction de compétences peut combiner la formation formelle à différents niveaux avec la promotion de l'apprentissage basé sur l'expérience.

- Le «construction de compétences » s'associe également à la forte présence de capital étranger dans l'agriculture et l'exploitation de matières premières. On peut y voir ici une réelle source d'apprentissage technologique basée sur la capacité d'absorption des connaissances étrangères et la capacité à négocier avec les principaux investisseurs étrangers.

- Enfin, les liens entre le secteur formel à forte valeur ajoutée et le secteur informel sont essentiels pour la « construction de compétences » des économies du Sud. Le rôle du secteur informel peut être vu comme une importante source d'innovation. Les activités informelles peuvent en ce sens être influencées par l'utilisation des technologies de l'information et de la communication.

Les S.N.I. ne sont plus uniquement perçus comme des instruments relatifs aux performances technologiques, mais bien comme des instruments utilisés pour comprendre le développement économique [LUN, 02]. L'effet de l'inclusion des activités d'innovation et des besoins (et aspirations) des populations dans l'analyse des performances en matière de développement économique est un domaine de recherche émergeant [HEE, $14 ; \mathrm{JOH}, 12]$. Ce thème a jusqu'ici été largement ignoré dans les études sur l'innovation systémique et le développement [COZ, 14]. En effet, la plupart des recherches empiriques sur l'inclusion et l'innovation se concentrent sur les processus microéconomiques (par exemple, les innovations frugales), mais l'importance des structures institutionnelles où les divers processus sont intégrés est largement méconnue [AND, 15, COZ, 14].

En 2012, un Rapport initié par le Réseau Globelics [JOH, 12] mettait en lumière la définition de l'innovation inclusive. Selon ce Rapport, l'innovation inclusive est un ingrédient du développement inclusif. Il s'agit de l'innovation par et pour les pauvres. Les populations défavorisées ont besoin de bons produits à bas prix. En cela, les entrepreneurs de base peuvent répondre aux besoins du bas de la pyramide grâce à une connaissance particulièrement fine de ce besoin. L'innovation systémique inclusive requiert trois particularités [JOH, 12] :

- L'importance des capacités existantes, menées par des processus d'apprentissage internes et externes à l'entreprise.

- L’importance des connaissances tacites, non codifiées et indigènes.

${ }^{3} \mathrm{Cf}$. www.globelics.org. II s'agit d'une large communauté scientifique travaillant sur l'innovation et la construction de compétences dans un contexte de développement économique. Son objectif principal est de contribuer à la création de connaissances académiques dans une perspective d'innovation et de développement. 
- L'importance des relations et des interactions dans le système social.

Le développement inclusif est un processus de changement structurel qui prend en considération les aspirations sociétales (collectives ou individuelles). Il redistribue les revenus générés dans les secteurs formels et informels et leur permet de façonner la société future en interaction avec d'autres groupes. On considère alors le «mal développement » non plus comme une phase particulière de l'état d'une économie donnée, mais comme un processus historique étudié à travers d'un ensemble d'éléments structurels mis dans leur contexte global. Cette perspective appelle à la construction de systèmes d'innovation qui prennent en compte la nature spécifique, sociale et les contextes spécifiques des économies en question.

L'innovation inclusive montre en quoi le développement des technologies couplé avec les politiques sociales visant à réduire les inégalités, sont des leviers importants dans les économies en développement (Lundvall, Lema, 2014). L’inclusion peut être «passive» ou «active » selon le degré d'implication des individus ou des communautés dans le développement économique. En ce sens, Arocena et Sutz (2003) montrent que les politiques d'innovation se traduisent par des politiques «bricolées » dans certains P.E.D. (latino-américains). Le potentiel scientifique et technique est écarté et les décideurs publics tricotent des mesures sans allouer de réelles ressources et moyens. Pour ces auteurs, la réalisation des politiques d'innovation dépend de la manière dont les coordinations (économiques et sociales) génèrent de l'innovation et de l'apprentissage. La dynamique «bottom-up » est primordiale pour comprendre les articulations entre les acteurs et les besoins des consommateurs, mais ces politiques doivent également s'orienter «top-down» en prévision d'objectifs économiques et sociaux bien définis et anticipés [AGU, 17].

\subsection{L'importance des universités dans les S.N.I. en développement}

Selon l'approche large des systèmes d'innovation, un certain nombre de travaux a formalisé l'entrée des systèmes éducatifs dans l'étude des S.N.I. [LUN, 99]. Parmi les travaux dans la littérature des S.N.I., on citera le rôle des systèmes éducatifs et des marchés du travail [LAM, 98], celui du management des ressources humaines dans les organisations [TOM,02] ou bien l'utilisation de la R\&D dans les firmes et établissements publics et/ou privés [LAL,00]. Le rôle des universités a par contre été plutôt écarté avant d'être remis plus récemment dans les débats actuels sur les S.N.I. [LUN,07; BRU, 09 ; CON, 02 ; ALB, 15].

Le système universitaire dans les S.N.I. du Sud est pourtant fondamental sur un double aspect : il place les diplômés dans les systèmes productifs tout comme il fournit le personnel en R\&D à travers la recherche fondamentale. Les relations université-entreprise peuvent également favoriser la création d'entreprises issues de la recherche universitaire [BRU, 09; DIE, 19]. Les collaborations entre universités et entreprises facilitent l'intégration des chercheurs au sein des entreprises à partir de la création de projets communs et permettent également une meilleure connaissance en matière de formation professionnelle. Les universités créent des diplômes de plus en plus professionnalisants permettant une meilleure insertion sur le marché du travail. L'objectif des universités est de faire émerger des liens avec le secteur industriel à travers des flux d'informations, de connaissances et de main-d'œuvre qualifiée.

Dans les P.E.D., un certain nombre de caractéristiques sont présentes [CAS, 14]: les entreprises des secteurs concurrentiels sont davantage engagées dans des dépenses liées à la $R \& D$ interne plutôt qu'à la R\&D externe; elles privilégient les sources internes pour innover; seule une petite proportion d'entreprises considère les universités comme une importante source d'innovation, d'informations, de connaissances et de compétences.

Dans les pays du Sud, un besoin de stimuler l'innovation dans les secteurs à faible valeur ajoutée est nécessaire afin de créer des trajectoires permettant de résoudre les problèmes, dans des conditions évidentes de pénurie [SCR,08]. Bien évidemment, un certain nombre de tendances sont à 
considérer sous l'angle de cette problématique. On citera d'abord la tendance à la privatisation de l'enseignement supérieur dans ces pays. La privatisation est l'une des réponses au manque de ressources dans certaines économies du Sud. Cette privatisation s'est réalisée avec une perte de qualité de l'enseignement. Un phénomène croissant de partenariats universitaires ou de filiales d'universités étrangères accompagne ce processus de privatisation de l'enseignement supérieur. Une seconde tendance est liée à la faiblesse des liens universités/industries. Les universités sont importantes dans les activités industrielles notamment dans les pays où la structure productive reconnait le rôle économique de la connaissance comme ressource créative et mercantile. Les compétences des diplômés sont primordiales dans un contexte de changement organisationnel et technique. Elles sont surtout importantes dans un contexte où la capacité d'absorption en lien avec les nouvelles technologies est un défi majeur [ALB,15].

Le phénomène de brain drain (fuite des cerveaux) et la mobilité des étudiants sont également des problèmes récurrents dans les pays en développement. La situation des «cerveaux » a fait couler beaucoup d'encre depuis une vingtaine d'années. La situation apparaît toujours comme alarmante. Si cette tendance est mondiale, elle est nettement plus perceptible sur le continent africain. Les Etats-Unis et la France constituent les principales destinations des cerveaux en fuite. Ce phénomène constitue une grande perte dans le sens où les pays pauvres enrichissent les universités des pays riches puisqu'ils fournissent, presque gratuitement car déjà formée, une expertise de pointe et souvent expérimentée. Enfin, une dernière tendance très marquée dans les P.E.D. est la faible demande de personnel très qualifié. Cette faible demande de diplômés est le reflet de barrières culturelles qui restreignent leur embauche. Cette fragile demande de compétences et connaissances peut créer une réelle barrière à l'innovation et au développement. Les politiques éducatives et les politiques industrielles et d'innovation ne sont pas coordonnées ; de ce fait, les universités dans les P.E.D. sont particulièrement vulnérables : privatisation, faible qualité de l'enseignement public, infrastructures dégradées, faibles salaires d'enseignants-chercheurs. Les arguments sont nombreux pour faire état de leur détérioration malgré leur rôle primordial dans le développement économique.

\subsection{Les politiques d'innovation au cœur des S.N.I. en développement}

$\mathrm{Si}$ les termes de politique scientifique et technologique génèrent beaucoup de travaux ces dernières années dans les pays industriels [BOR, 13], ils ont été plus rares dans l'orientation des politiques publiques des économies du Sud. Or il n'est pourtant plus à reconnaitre que la science et la technologie sont considérées par de nombreux auteurs comme les socles des mécanismes de développement et de la croissance permettant potentiellement de réduire les inégalités mondiales [DOG,09]. Il est ainsi observé plusieurs évolutions conceptuelles des politiques publiques liées à la science, la technologie et l'innovation (S.T.I.). Elles se différencient principalement en fonction de la conceptualisation même de l'innovation, préalablement détaillée [EDQ, 04]. Les premières concernent les politiques technologiques qui s'intéressent aux infrastructures et au développement technologique promouvant la $\mathrm{R} \& \mathrm{D}$. Les secondes, plus indirectes, prennent en considération la formation du capital humain par l'éducation, l'enseignement supérieur, la formation professionnelle [GOU, 15]. Elles réhabilitent parfois la contribution de travaux plus anciens sur la notion de capacités d'apprentissage [CRO, 97]. Plus largement, tous les instruments qui influencent l'innovation doivent être pris en compte (ceux liés au nouveau produit ou processus, mais également à l'ensemble des processus, de la création de nouveaux produits aux moyens de les utiliser pour mieux les diffuser). Dans cette optique, la politique d'innovation a pour objectif de contribuer à la prospérité et au bienêtre économiques [FAG, 11].

Tout comme sa conceptualisation, deux grands ensembles d'instruments sont complémentaires dans le développement de politiques d'innovation : les instruments qui influencent directement les politiques industrielles et technologiques $[\mathrm{PAD}, 14]$ et ceux plus indirectement liés à l'innovation [FAG,17 ; CAS, 17]. Parmi les instruments directs, on recense trois sous-ensembles : tout d'abord le cadre institutionnel et les politiques générales de promotion des sciences, technologies, industries. 
Ils incluent la création d'organisations publiques telles que des secrétariats, des conseils et des ministères. Ils passent plus concrètement par l'élaboration de plans nationaux, régionaux et sectoriels ou de mécanismes de coordination entre les différents niveaux gouvernementaux. Ils peuvent prendre la forme de programmes favorisant le développement et l'innovation technologiques, la création d'organismes publics pour protéger la propriété intellectuelle ou la mise en œuvre de politiques de normalisation et de qualité des actions.

Un deuxième groupe d'instruments est axé sur le financement. Il peut s'agir d'incitations fiscales spécialement conçues pour la $R \& D$. Ce financement passe également par des subventions publiques directes, telles que des subventions pour des secteurs ou des régions en particulier. Les autres instruments financiers peuvent prendre la forme de subventions, garanties de prêt public, crédits bonifiés, fonds publics de capital-risque et soutien à la commercialisation de l'innovation.

Un troisième ensemble d'instruments vise à promouvoir une plus grande interaction entre les acteurs du système et à diffuser les connaissances technologiques. Des programmes favorisant la recherche conjointe public-privé, les échanges de personnel ou les détachements entre universités et entreprises, ainsi que la mobilité des chercheurs peuvent être mis en œuvre pour favoriser ces interactions. L'interaction peut également être encouragée par le biais de services de transfert de technologie opérant dans des universités. Un dernier instrument est le lancement d'initiatives visant à promouvoir l'importance et l'utilité des politiques d'innovation (séminaires, conférences et activités qui encouragent la culture de l'innovation parmi les entrepreneurs et les étudiants).

De l'autre côté, on observe un ensemble des politiques indirectement liées aux processus d'innovation et de R\&D mais dont leur mise en place est prioritaire dans les économies du Sud [CAS,17]. Ces politiques peuvent être de différentes natures: éducatives, de santé, environnementales... Elles peuvent être appréhendées à des échelles nationales ou au niveau des collectivités territoriales. Ces dernières influent sur les politiques d'innovation qui, à leur tour, sont relayées au premier plan des processus d'apprentissage. Leur efficacité nécessite une coordination étroite à un certain nombre de niveaux / domaines différents, ainsi que la mise au point de nouvelles formes de gouvernance et de bases de connaissances complémentaires qui rendent cela possible. Mais ce challenge s'avère très difficile, notamment dans les P.E.D. Sa réussite dépend fondamentalement de la capacité du système politique d'un pays (et de son gouvernement) à traiter ses propres problèmes. Dès lors, il est nécessaire de prêter attention aux expériences tentées par différents P.E.D. pour atteindre ces objectifs, dans le but d'identifier les facteurs qui influencent les trajectoires permettant de tirer le meilleur parti possible des politiques d'innovation [FAG,17].

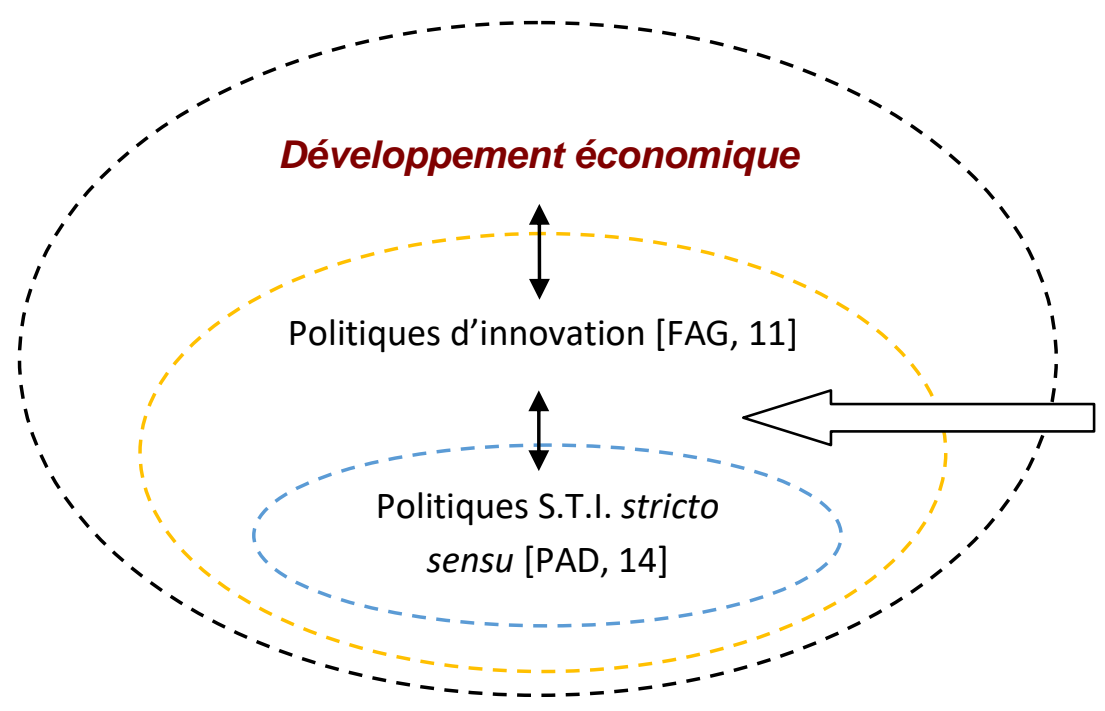

Instruments :

Directs :

Cadre institutionnel et

politiques générales de S.T.I.

Financement, capital risque

Promotion des interactions S.T.I.

Indirects :

Politiques éducatives, sociales, environnementales 


\section{Conclusion}

Un système d'innovation décrit les relations entre institutions (scientifiques, technologiques, industrielles, commerciales, financières, politiques), privées et publiques (entreprises, laboratoires de recherche et d'ingénierie, administrations...). Ces relations sont constituées par des flux financiers, cognitifs, informationnels, matériels et humains. Le but d'un tel système est de «produire » des innovations. Ce système peut être observé et étudié sur les plans sectoriel, territorial ou entrepreneurial, mais dès que l'on se réfère aux dispositifs de coordination, de valorisation et de régulation, le champ de l'étude s'étend au plan national. Une analyse à partir du système (national) d'innovation permet d'étudier l'environnement des entreprises pour comprendre leur tendance à innover et à se transformer. La nature systémique des relations qui caractérisent un S.N.I. explique en effet ce qui favorise ou non l'acte d'innover. Mais, doit-on réduire l'innovation, produit du système, aux seuls échanges inter-organisationnels débouchant sur une nouvelle combinaison productive ? Résulte-t-elle uniquement d'une organisation spécifique des relations économiques ? Notre article montre que la systémique des liaisons entre les acteurs économiques et sociaux ne se réfère pas uniquement aux interactions économiques, cognitives et technologiques mais prend aussi en considération les structures sociales qui sont à l'origine de ces comportements innovateurs. Or, les institutions (État et collectivités locales) tiennent un rôle significatif dans l'organisation et l'évolution des structures socio-économiques. En retour le S.N.I. (au sens large ou décliné aux niveaux territorial ou sectoriel) contribue à la performance innovante des entreprises par l'offre des ressources scientifiques et techniques qu'il peut organiser [CAS,15].

Concept flexible et adaptatif, le S.N.I. étend sa structure conceptuelle selon le champ empirique visité. Il est ainsi assez flexible pour se conformer aux spécificités des pays en développement [LAS,03]. Il s'affirme donc comme un instrument pilote d'analyse des perspectives de croissance et de développement des économies en développement tout comme celles des pays développés. Au total, il évolue à travers une variété de chemins dépendant de l'équilibre des forces en présence : il peut apparaître comme le garant de la croissance et des performances économiques tout en favorisant la cohésion sociale, aussi bien qu'il est susceptible de créer ou d'atténuer les disparités de revenus [MUC,04].

A la lumière de ces éléments, il semble notoire que les pays ayant mis en lumière la diversité des savoirs tout en valorisant le rôle des universités, par des politiques d'innovation fiables, ont su construire un S.N.I. viable. De plus, de par les incitations et la formation d'un ensemble cohérent de règles et de politiques, la formation de S.N.I. sera d'autant plus pérennisée. Certains pays émergents ont ainsi réussi ce pari malgré certaines faiblesses structurelles encore visibles [CAS,17]. Entre autres, quelques exemples de pays émergents: en Indonésie, l'insuffisance des collaborations entre recherche et industrie, couplée avec celle de la R\&D limitent la production d'innovation et l'absorption de technologies. Au Nigéria, l'absorption de technologies n'est pas prioritaire et indirectement dépendante de la mise en place d'un plan de redressement, basé sur la compétitivité, la protection sociale, la gouvernance et la sécurité. En Turquie, très dépendante de l'extérieur, la politique en faveur de l'innovation des entreprises a ciblé des programmes de recherche et développement ambitieux qui, malgré une économie résiliente, n'ont pas encore porté leurs fruits.

Chaque pays a son propre modèle de construction systémique d'innovation, porté par son contexte culturel, historique et sa structure socio-économique. Il est d'ailleurs important de rappeler que cette construction de savoirs s'établit à tous les niveaux : individuel (microéconomique), collectif (mésoéconomique) et au sein de la structure globale de l'économie (macroéconomique). Cette triple échelle rappelle la définition la plus communément admise d'un système national d'innovation reposant à la fois sur la diversité des sources d'apprentissage pour l'entreprise innovante, l'organisation du travail inter et intra-entreprises, la structure économique du pays et sa structure sociale représentée par ses liens sociaux [CHA,18]. 


\section{Bibliographie}

[AGH,98] AGHION P., HOWITT P., Endogenous Growth Theory, MIT Press, Cambridge, 1998.

[AGU,17] AGUIRRE-BASTOS C., WEBER M., Foresight for shaping national innovation systems in developing countries, Technological Forecasting and Social Change, 128, p. 186-196, 2017.

[ALB,15] ALBUQUERQUE E., WILSON S., GLENDA K., KEUN L., Developing National Systems of Innovation: University-Industry Interactions in the Global South, Cheltenham, Edward Elgar Publishing \& IDRC, 2015.

[ALT,09] ALTENBURG T., Building inclusive innovation systems in developing countries: challenges for IS research, dans LUNDVALL B.A., JOSEPH K.J, CHAMINADE C., VANG J. Handbook of Innovation systems and developing countries, Elgar Publishing, Cheltenham, p. 33-57, 2009.

[AND,15] ANDERSEN A.D, JOHNSON B., Low carbon development and inclusive innovation systems, Innovation and Development, 5(2), p. 279-296, 2015.

[ARO,03] AROCENA R., SUTZ J., Understanding underdevelopment today: news perspectives on NSI, Global Network for Economic of Learning, Innovation and Competence Building Systems, Brésil, 2003.

[BEL,07] BELL M., Technological learning and the development of production and innovative capacities in the industry and infrastructure sectors of the least developed countries: what role for ODA?, Report 2007, Unctad, Genève, 2007.

[BEL,96] BELLON B., NIOSI J., Les systèmes nationaux d'innovation et la formation scientifique et technique, in Tremblay D., Innovation Technologique et Qualification, Presses de l’Université du Québec, 1996.

[BOR,13] BORRAS S., EDQUIST C., The choice of innovation policy instruments, Technological forecasting and social change, p. 1513-1522, 2013.

[BRA,98] BRANSCOMB L.M., KELLER J.H., Investing in Innovation: Creating a Research and Innovation Policy that Works, The Journal of Technology Transfer, 25(2), p. 247-247, 1998.

[BRU,11] BRUNDENIUS C., LUNDVALL B.A., SUTZ J., The role of universities in innovation systems in developing countries: developmental university systems - empirical, analytical and normative perspectives, in LNDVALL B.A, JOSEPH K.J, CHAMINADE C., Handbook of innovation systems and developing countries, Elgar Publishing, Cheltenham, 2011.

[CAS,11] CASADELLA V., Le SNI dans les PED, Editions Universitaires Européennes, 2011.

[CAS,15] CASADELLA V., LIU Z., UZUNIDIS D., Innovation capabilities and economic development in open economies, ISTE Ltd, Wiley and Sons, New York, 2015.

[CAS,17a] CASADELlA V., TAHI S., Capacités et politiques d'innovation dans les pays moins avancés : enseignements tirés du cas du Sénégal, Innovations, 53(2), p. 13-39, 2017.

[CAS,17b] CASADELLA V., UZUNIDIS D., National Innovation Systems of the South, Innovation and Economic Development Policies: A Multidimensional Approach, Journal of Innovation Economics \& Management, 23,(2), p. 137-157, 2017.

[CAS,03] CASSIOLATO J.E., LASTRES H.M., MACIEL M.L., Systems of innovation and development: Evidences from Brazil, Cheltenham, Edward Elgar, 2003.

[CAS,14] CASSIOLATO J.E., MATOS M.P., LASTRES H.M., Innovation systems and development, Currie-Alder B., Kanbur R., Malone D.M, International development: ideas, experience and prospects, Oxford University Press, 2014.

[CHA,18] CHAMINADE C., LUNDVALL B.A., HANEEF S., Advanced introduction to national innovation systems, Cheltenham, Edward Elgar, 2018.

[COH,99] COHENDET P., FORAY D., GUELLEC D., MAIRESSE J., (1999), La gestion publique des externalités positives de recherche, dans FORAY D., MAIRESSE J., Innovations et Performances: Approches Interdisciplinaires, Paris, Edition de l'EHESS, p. 367-383.

[CON,02] CONCEICAO P., HEITOR M.V (2002), Knowledge interaction towards inclusive learning: Promoting systems of innovation and competence building, Technological Forecasting and Social Change, 69, p. 641-651, 2002.

[COZ,14] COZZENS S., SUTZ J., Innovation in informal settings: reflections and proposals for a research agenda, Innovation and Development, 4(1), p. 5-31, 2014. 
[CRO,97] CROZIER M., FRIEDBERG E., L'acteur et le système, les contraintes de l'action collective, Paris, Points, 1997.

[DIE,19] DIE, D. M., Les traits caractéristiques de la relation université-entreprise au Cameroun, Marché et Organisations, 34(1), p. 133-157, 2019.

[DOG,09] DOGSON M., Asia's national innovation systems: institutional adaptability and rigidity in the face of global innovation challenges, Asia Pacific Journal of Management, 26(3), p. 589-609, 2009.

[EDQ,01] EDQUIST C., The system of innovation approach and innovation policy: an account of the state of the art, DRUID Conference, Aalborg School, Danemark, 2001.

[EDQ,04] EDQUIST C., Systems of innovation: a critical review of the state of the art, Handbook of Innovation, Oxford University Press, 181-2008, 2004.

[FAG,11] FAGERBERG J., FOSAAS M., BELL M., MARTIN B.R, Christopher Freeman: Social science entrepreneur, Research Policy, 40, p. 897-916, 2011.

[FAG,15] FAGERBERG J., Innovation policy, national innovation systems and economic performance: in search of the useful theoretical framework, EU-SPRI conference "Science and innovation policy: Dynamics, Challenges, Responsibility and Practice", Manchester, UK, 18-20 June, 2015.

[FREE,87] FREEMAN C., Technology Policy and Economic Performance: Lessons from Japan, Londres, Pinter, 1987.

[FREE,88] FREEMAN C., Japan a new national system of innovation?, in Dosi G. et al. (eds), Technical Change and Economics Theory, Londres, Pinter, p.330-348., 1988.

[FREE,95] FREEMAN C., The 'national system of innovation' in historical perspective, Cambridge Journal of Economics, 19(1), p.5-24., 1995.

[GOD,04] GODARD O., Savoirs, risques globaux et développement durable, Cahier de Recherche, n²004-003, Chaire développement durable EDF-Ecole Polytechnique, 2004.

[GOU,15] GOULET F., COMPAGNONE C., LABARTHE P., Émergence des conseillers privés du conseil en agriculture : de nouvelles interrogations pour la recherche, dans GOULET F., COMPAGNONE C., LABARTHE P., Conseil privé en agriculture : acteurs, pratiques, marché, Versailles, Quae, p. 201-216., 2015.

[GUE, 03] GUESNERIE R., HAUTCOEUR P., La Recherche au Service du Développement Durable - Rapport Intermédiaire, Paris, Ministère de la Recherche et des Nouvelles Technologies, 2003.

[HEE,14] HEEKS R., FOSTER C., NUGROHO Y., New models of inclusive innovation for development, Innov. Dev., 4 (2), 175-185, 2014.

[HOB,95] HOBDAY M., Innovation in East Asia: the challenge to Japan, Cheltenham, Edward Elgar, 1995.

[HOB,04] HOBDAY M., RUSH H., BESSANT J., Approaching the innovation frontier in Korea: the transition phase to leadership, Research Policy, 33(10), 1433-1457, 2004.

[JEN,07] JENSEN M.B., JOHNSON B., LORENZ E., LUNDVALL B.A, Forms of Knowledge and Modes of Innovation, Research Policy, 36(5), p.680-693, 2007.

[JOH,12] JOHNSON B., ANDERSEN A.D, Learning, Innovation and Inclusive Development: New Perspectives on Economic Development Strategy and Development Aid, University Press, Aalborg, 2012.

[JOH,03] JOHNSON B., LUNDVALL B.A., National System of Innovation and Economic development, in Muchie M., Gammerltoft P., Lundvall B.A., Putting Africa First: the making of African Innovation Systems, Aalborg University Press, p.13-29, 2003.

[KIM,97] KIM L., Imitation to innovation: The dynamics of Korea's technological learning, Boston MA, Harvard University Press, 1997.

[JUM,01] JUMA C.F., HONCA D., HUETE-PEREZ J., KONDE V., LEE S., Global governance of technology: meeting the needs of developing countries, International journal of technology management, 22(7-8), 2001.

[KLI,86] KLINE S.J., ROSENBERG N., An overview of innovation, in Rosemberg N., Landau R., (eds), The positive sum strategy: harnessing technology for economic growth, Washington, National Academy Press, p. 275-305, 1986. 
[LAL,00] LALL S., Technological Change and Industrialization in the Asian Newly Industrializing Economies: Achievements and Challenges, dans KIM L., NELSON R., (eds), Technology, Learning and Innovation, Cambridge University Press, p. 13-69, 2000.

[LAM,98] LAM A., Tacit knowledge, Organisational learning and innovation: a societal perspective, DRUID Working Paper Nº98-22, Aalborg School, 1998.

[LIS,57] LIST F., "Système National d'Economie Politique”, Première édition française, Paris : Capelle. Réed. (1998), Paris: Gallimard, 1857.

[LUN,85] LUNDVALL B.A Product Innovation and User-Producer Interaction, Aalborg, Aalborg University Pres, 1998..

[LUN,88] LUNDVALL B.A, Innovation as an interactive process: from user-producer interaction to the national system of innovation, in DOSI G. et al.,. (eds.), Technical Change and Economic Theory, Londres, Pinter, p. 349369, 1998.

[LUN,92] LUNDVALL B.A., National Systems of Innovation: Towards a Theory of Innovation and Interacive Learning, Londres, Pinter, 1992.

[LUN,02] LUNDVALL B.A., The Learning Economy: challenges to economic theory and policy, A Modern Reader in Institutional and Evolutionary Economics: Key Concepts, Cheltenham, Edward Elgar, p. 26-47, 2002.

[LUN,07] LUNDVALL B.A, Innovation systems: theory and policy, Elgar Companion to Neo-Schumpeterian Economics, Cheltenham, Edward Elgar, 2007.

[LUN,13] LUNDVALL B.A, Innovation Studies: a personal interpretation of the state of the art, in Fagerberg J., Martin B., Andersen E. (eds.), Innovation Studies, Evolution and future challenges, Oxford, Oxford University Press, p.21-71, 2013.

[LUN,99] LUNDVALL B.A, CHRISTENSEN J.L. Extending and deepening the analysis of innovation systems- with empirical illustrations from the DISCO project, DRUID Working Paper N 99-12, Aalborg University, 1999.

[LUN,94] LUNDVALL B.A, JOHNSON B., The Learning Economy, Journal of Industry Studies, 1(2), p. 23-42, 1994.

[LUN,02] LUNDVALL B.A., JOHNSON B., ANDERSEN E.S, DALUM B., National systems of production, innovation and competence building, Research Policy, 31(2), p. 213-231, 2002.

[LUN,14] LUNDVALL B.A, LEMA R., Growth and structural change in Africa: development strategies for the learning economy, African Journal of Science, Technology, Innovation and Development, 6(5), p. 455-466, 2014.

[MCC,99] MC CORMICK D., African Enterprise Clusters and Industrialization: theory and reality, World Development special issue on enterprise clusters, 27(9), p. 1531-1551, 1999.

[MET,94] METCALFE S.. (1994), Evolutionary economics and technology policy, The Economic Journal, 104, p. 931-944, 1994.

[MOH,05] MOHNEN L., ROLLER L., Complementarities in innovation policy, European Economic Review, 49, p. 1431-1450, 2005.

[MUC,04] MUCHIE M., A theory of an Africa as a unification nation: a re-thinking of the structural transformation of Africa, African Sociological Review, 8(2), p. 136-157, 2004.

[MYT,03] MYTELKA L., The dynamics of catching-up: the relevance of an innovation system approach in Africa, dans MUCHIE M., GAMMERLTOFT P., LUNDVALL B.A., . (eds.), Putting Africa First: the making of African Innovation Systems, Aalborg University Press, p. 29-43, 2003.

[MUC,13] MUChIE M., GAMMERLTOFT P., LUNDVAll B.A., Putting Africa First: the making of African Innovation Systems, Aalborg University Press, 2013.

[NAL,96] NALEBUFF B., BRANDEBURGER A., La Co-opétition, une Révolution dans la Manière de Jouer: Concurrence et Coopération, Paris, Village mondial, 1996.

[NAR,04] NARULA R., Understanding absorptive capacities in a Innovation Systems context: Consequences for Economic and Employment Growth, DRUID Working Paper N04-02, Aalborg School, 2004.

[NEL,88] NELSON R.R., Institutions supporting technical change in the United-States, in DOSI G. et al., Technical Change and Economic Theory, London, Pinter, p. 312-329, 1988. 
[NEL,93] NELSON R.R., National Innovation System: A Comparative Analysis, Oxford, Oxford University Press, 1993.

[NEL,82] NELSON R.R., WINTER S., An evolutionary theory of economic change, Amsterdam, Holland Press, 1982.

[NIO,93] NIOSI J., SAVIOTTI P., BELLON B., CROW M., National systems of innovation: in search of a workable concept, Technology and Society, 15, p. 207-227, 1993.

[OCD,99] OCDE, Gérer les systèmes nationaux d'innovation, Paris, OCDE, 1999.

[PAD,14] PADILLA-PEREZ R., GAUDIN Y., Science, technology and innovation policies in small and developing economies: The case of Central America, Research Policy, 43, p. 749-759, 2014.

[PEN,03] PENIN J., Endogénéisation des externalités de recherche: le rôle de la capacité d'émission des connaissances, Revue d'économie industrielle, 102, p. 7-28., 2003.

[SCR,08] SCRINIVAS S, SUTZ J., Developing countries and innovation: searching for a new analytical approach, Technology in society, 30(2), p.129-140, 2008.

[SEN,00] SEN A., Development as Freedom, Anchor Books, New York, 2000.

[SMI,00] SMITH K., Innovation as a systemic phenomenon: rethinking the role of policy, Enterprise \& Innovation Management Studies, 1(1), p.73-102, 2000.

[TEE,07] TEECE D., Explicating dynamic capabilities: The nature and microfoundations of (sustainable) enterprise performance, Strategic Management Journal, 28(12), 1319-1350, 2007.

[TOR,15] TORRE A., ZIMMERMANN J.B., Des clusters aux écosystèmes industriels locaux, Revue d'économie industrielle, 152(4), p. 13-38., 2015.

[UZU,10] UZUNIDIS D., PATELIS D., Le nouveau mercantilisme, la mondialisation et sa crise, Recherches internationales, 88, p. 19-40, 2010. 